December 2012

\title{
Radiation exposure in interventional procedures
}

G. K. Korir

University of Massachusetts Lowell

B. O. Ochieng

Aga Khan University

J.S. Wambani

Kenyatta National Hospital

I. K. Korir

National Nuclear Regulator

C. Y. Jowi

University of Nairobi

Follow this and additional works at: http://ecommons.aku.edu/ eastafrica_fhs_mc_imaging_diagn_radiol

Part of the Radiology Commons

\section{Recommended Citation}

Korir, G. K., Ochieng, B. O., Wambani, J. S., Korir, I. K., Jowi, C. Y. (2012). Radiation exposure in interventional procedures. Radiation Protection Dosimetry, 152(4), 339-344.

Available at: http://ecommons.aku.edu/eastafrica_fhs_mc_imaging_diagn_radiol/6 


\title{
RADIATION EXPOSURE IN INTERVENTIONAL PROCEDURES
}

\author{
G. K. Korir ${ }^{1, *}$, B. O. Ochieng 2 , J. S. Wambani ${ }^{3}$, I. K. Korir ${ }^{4}$ and C. Yuko Jowi ${ }^{5}$ \\ ${ }^{1}$ Department of Physics and Applied Physics, University of Massachusetts Lowell, One University Ave., \\ Lowell, MA 01854, USA \\ ${ }^{2}$ Heart \& Cancer Centre, Aga Khan University Hospital, PO Box 30270-00100, Nairobi, Kenya \\ ${ }^{3}$ Radiology Department, Kenyatta National Hospital, PO Box 20723-00202, Nairobi, Kenya \\ ${ }^{4}$ National Nuclear Regulator, Eco Glades 2 Office Park, Block G, Eco Park, Centurion 0157, South Africa \\ ${ }^{5}$ Department of Pediatrics and Child Health, University of Nairobi, PO Box 19676-00202, Nairobi, Kenya \\ *Corresponding author: chumo2009@gmail.com
}

Received January 2 2012, revised March 20 2012, accepted April 192012

The aim of this study was to estimate radiation doses patients and staff are exposed to during interventional procedures (IPs), compare them with the international diagnostic reference levels and to develop initial National Diagnostic Reference Levels. The IP survey was undertaken as the initial task of which, retrospective data were collected from the only four Kenyan hospitals carrying out interventional radiology and cardiology procedures at the time of the study. Real-time measurement of radiation dose to patients and staff during these procedures was done. To the patients, kerma-area product (KAP) and fluoroscopy time measurements were done using an in-built KAP meter, while peak skin dose (PSD) was measured using slow Extended Dose Range $\left(\mathrm{EDR2}^{\circledR}\right)$ radiographic films. The staff occupational doses were measured using individual thermo-

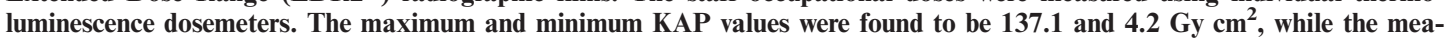
sured PSD values were 740 and $52 \mathrm{mGy}$, respectively. The fluoroscopic time range was between 3.3 and 70 min. The staff doses per procedure ranged between 0.05 and $1.41 \mathrm{mSv}$ for medical doctors, 0.03 and $1.16 \mathrm{mSv}$ for nurses, 0.04 and 0.78 $\mathrm{mSv}$ for radiographers and 0.04 and $0.88 \mathrm{mSv}$ for clinical staff. The measured patient PSDs were within the threshold limit for skin injuries. However, with the current few IP specialists, an annual increase in workload as determined in the study will result in the International Commission on Radiation Protection annual eye lens dose limit being exceeded by $10 \%$. A concerted effort is required to contain these dose levels through use of protective gear, optimisation of practice and justification.

\section{INTRODUCTION}

Interventional fluoroscopy procedures are medical procedures in which potentially high dose rate X-ray fluoroscopy with high temporal resolution may be used to guide the navigation, placement or manipulation of medical devices inside the human body. Although originally developed for radiologists, interventional radiology (IR) is now being used also by many other medical specialists - cardiologists, urologists, gastroenterologists, orthopaedic surgeons, cardiovascular surgeons, neurosurgeons, traumatologists, anaesthesiologists and paediatricians. Interventional procedures (IPs) are therefore divided into many categories, such as IR and interventional cardiology (IC). In most countries, irrespective of the level of healthcare provision, IPs offer the opportunity to diagnose and treat more patients with a wide range of pathological issues at a relatively lower cost. Compared with conventional surgery, interventional techniques do not require an expensive operating room, space for in-patient hospital admissions and risk associated with the use of local anaesthetics. The disadvantages of local anaesthesia are excessively high concentration of the anaesthetic in the blood, injury from the needle used to inject the drug and discomfort due to inadequate anaesthetic. Additionally, IPs allow biopsy of lesions that were previously inaccessible via other available means, at a relatively lower cost but with concomitantly greater risks ${ }^{(1)}$.

IPs have been extensively developed and may be classified into different types such as drainage, coil embolisation, filter placement, stenting and foreign-body retrieval, or into anatomical systems such as vascular, gastro-intestinal and urological procedures. Modern fluoroscopic systems that use image intensifiers coupled to closed-circuit television unit have encouraged the rise in the frequency of IPs over the years. Originally, fluoroscopy was carried out in a dark room with the radiologist viewing the faint scintillations from a thick fluorescent screen ${ }^{(2)}$.

Many modern catheterisation laboratories have improved in-built radiological protection measures and reduced scatter radiation. In addition, the inclusion of radiation protection training for interventional radiologists and cardiologists has played a role in the reduction of the risk of radiation exposure, even though the complexity and the number of procedures have increased ${ }^{(3)}$. Therefore, evaluation and follow-up of occupational doses should be considered an aspect of extra importance of the quality 


\section{G. K. KORIR ET AL.}

assurance programme. In a study of radiation-associated posterior lens opacities carried out in Serbia, a strong dose-response was found between occupational exposure and the prevalence of radiation-associated posterior lens changes ${ }^{(4)}$. Occupational exposure risk in IPs is higher than in other specialties that involve the use of X-ray. This is because the specialists performing the interventions monitor the procedures on a real-time basis by staying close to the patients who are being exposed to radiation. Radiation exposure is, therefore, a significant concern in this type of IPs and is aggravated further by the low number of specialists, who thus experience large workloads and associated complex procedures ${ }^{(5)}$.

The use of interventional fluoroscopy is increasing in Kenya, as evident from the rise in the number of hospitals performing IPs from two in 2000 to five in 2010 as well as from the workload. It is likely that many non-radiologist practitioners will soon join the interventionists' fray, increasing the projected scope of IPs. Most of these interventionists have little or no relevant safety knowledge and experience. Some patient dose studies carried out in the UK have reported the mean peak skin dose (PSD) that exceeds the 2 Gy thresholds for deterministic effects in $2 \%$ of the cases ${ }^{(6)}$.

Diverse methods of assessing patient dose exist worldwide. Modern fluoroscopic systems have an inbuilt ion chamber (KAP meter) that measures the total X-ray energy leaving the X-ray tube. Kermaarea product (KAP) gives a reasonable estimate of the dose measurement of any procedure that is used. PSD is the highest radiation dose (entrance surface air kerma) at any portion of a patient's skin during a procedure. As of 2010, the commercially available fluoroscopic unit capable of calculating or displaying PSD was not commonly available ${ }^{(7)}$; however, use of films was appropriate for this study because of the indication of the likelihood of deterministic effects.

Currently, Kenya has no data on patient dose resulting from IPs as well as dose to the staff involved in the process of carrying out these procedures. Evaluation of risks posed by the radiation that patients and staff in IPs are exposed to is complicated because of diversity in examination and personnel involved. This situation creates a major challenge in implementing radiation safety measures. Consequently, this study was initiated in 2007 to assess the level of radiation exposure at the only four hospitals carrying out IPs in Kenya. The results were compared with the national reference doses for the $\mathrm{UK}^{(8)}$ and the International Commission on Radiation Protection (ICRP) occupational dose limits ${ }^{(9)}$, thereby providing the baseline data to be used in sensitising IP personnel to the level of radiation they are exposed to in their daily practice and its risks and to how to justify and optimise radiation safety measures.

\section{MATERIALS AND METHODS}

\section{IPs statistics}

A questionnaire-type form was developed to record patient information, IP statistics and parameters associated with the fluoroscopic equipment.

\section{Patient dose assessment}

Slow radiographic films (Kodak EDR2 ${ }^{\circledR}$ ) with a measuring range of 0.01 to $\sim 2$ Gy was used for 54 randomly selected IPs and PSD measurements. The films indicate the distribution of dose and skin locations where the dose measurements exceed the limit for deterministic effects. The films were calibrated by segmenting them into four portions and each portion was exposed to a known radiation dose separately while shielding the other three with lead shields. The optical density (OD) was measured using a X-Rite ${ }^{\mathbb{Q}}$ model $341 \mathrm{C}$ transmission densitometer (X-Rite Inc., USA) on films exposed to the known dose of 0.01 to $\sim 1 \mathrm{~Gy}^{(10)}$. The Kodak EDR2 ${ }^{\circledR}$ OD calibration curve was then plotted against the known dose to generate a calibration curve. The curve was fitted to generate a secondorder polynomial equation as follows

$$
\begin{aligned}
y= & 1 \times 10^{-8} x^{3}-2 \times 10^{-5} x^{2}+0.0132 x \\
& +0.2106
\end{aligned}
$$

where $y$ and $x$ represent OD and relative dose in $\mathrm{mGy}$, respectively.

Measurements of individual patient doses were carried out using Kodak EDR2 ${ }^{\circledR}$ films and the machine's in-built KAP meter. The film was placed under the patient table and positioned at the central beam of the patient body's region of interest. The exposed films were processed at least $1 \mathrm{~h}$ after exposure to allow the densities to stabilise. The OD values measured were used to obtain PSD, using Equation (1). $\mathrm{kVp}$, tube loading (mAs), KAP reading and fluoroscopic time were all recorded for each procedure. The KAP meter was reset to zero before the commencement of each procedure and the cumulative KAP values were recorded at the end of the procedure. The third quartile values per examination type were considered as the initial National Diagnostic Reference Levels (NDRLs) for KAP and fluoroscopy time, respectively.

\section{Staff dose measurement}

A total of 216 personnel were involved in the 54 IPs assessed in the study. All these personnel namely medical doctors (MDs), nurses, radiographers and clinical staff were provided with thermoluminescence dosemeters (TLDs) (lithium fluoride TLD 100; 
Harshaw-Filtrol, Cleveland) prior to each procedure. The TLDs were clipped on their collars. These TLDs were in addition to the ones used for routine monthly personal monitoring. After each procedure, the doses recorded by the TLDs were read out using a TLD Reader (Harshaw Model 4500) operating with the WinREMS software. These readings were corrected for the natural background level readings recorded by the control TLDs.

\section{RESULTS}

\section{IPs statistics}

The total annual IP workload for all the four facilities combined was found to be 1029 patients, which consisted of $75 \%$ adults and $25 \%$ children. The adult procedures were predominantly coronary angioplasty (CA) and aortic lesion for diagnostic procedures, while the therapeutic IPs were CA and stenting. The most common paediatric cardiology procedures include diagnostic cardiac catheterisations for shunt assessment such as ventricul-septal defects associated with pulmonary hypertension, tetrology of Fallot and its clinical variants and other complex congenital cardiac catheterisations such as transposition of great vessels and the dextrocardia. Therapeutic paediatric IPs which were done included pulmonary vasculature for congenital heart disease, patent ductus ateriosus, embolisation and mitral vulvoplasty for acquired heart disease. The increasing rates per annum were roughly 30 and $40 \%$ for adult and paediatric patients, respectively. The IPs were distributed as follows: diagnostic procedures $49 \%$, therapeutic procedures $21 \%$ and combined diagnostic and therapeutic procedures $30 \%$. The average annual workload per medical specialist was 130 IPs $(10 \%)$ with the leading specialist performing 330 IPs (25\%). IC comprised $87 \%$ with adult CA accounting for $33 \%$, paediatric CA $12 \%$ and paediatric valvoplasty $42 \%$. Interventional neuro-radiology comprised $13 \%$; most of which were four-vessel angiography. While 1029 indicated the total annual number of IPs obtained retrospectively, the data points in Figures 1-5 reflect empirical values obtained by the actual measurements of patient and occupational doses. Figure 1 shows the distribution of the 54 randomly selected adult and paediatric patients who underwent IPs and the study parameters measured. There were $25 \%$ of complex procedures carried out in $>30 \mathrm{~min}$. The fluoroscopic time in IC procedures was distributed as follows: $3 \%$ took $<10 \mathrm{~min}, 27 \%$ took between 10 and $20 \mathrm{~min}$ and $21 \%>20 \mathrm{~min}$. In interventional neuro-radiology procedures, $10 \%$ took $<10 \mathrm{~min}, 2 \%$ between 10 and $20 \mathrm{~min}$, while $4 \%$ took $<20 \mathrm{~min}$.

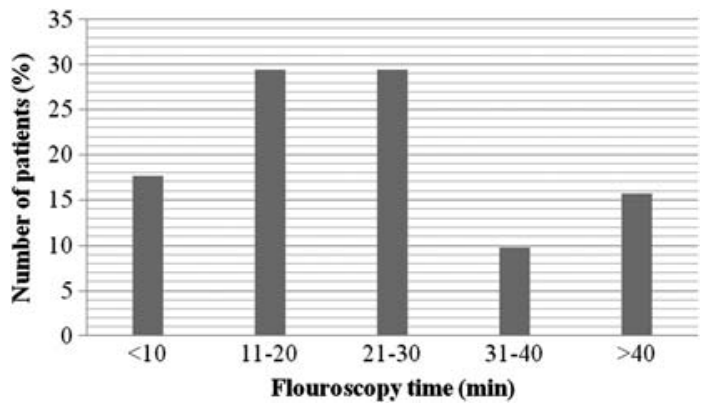

Figure 1. Distribution of the complexity of procedures according to time spent.

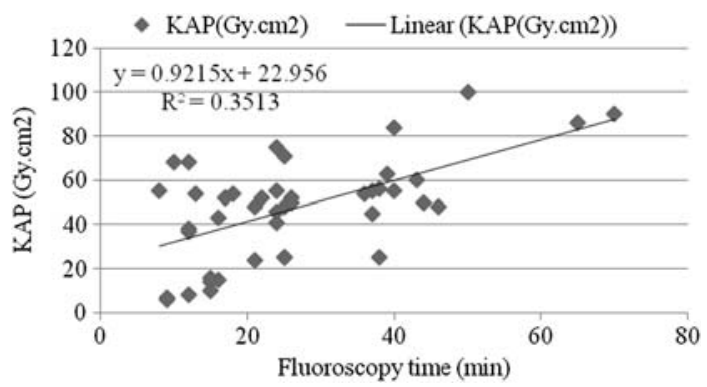

Figure 2. Distribution of the correlation between KAP and fluoroscopy time measurements for the examinations considered.

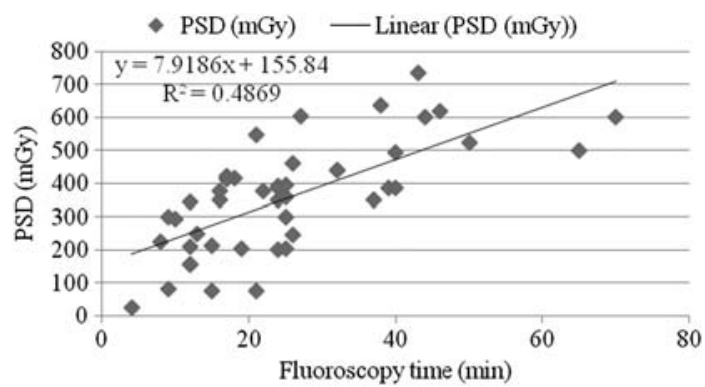

Figure 3. Measurement correlation between PSD and fluoroscopic time.

\section{Patient dose}

Figure 2 shows the distribution of KAP and the fluoroscopy time for the examinations considered. The average KAP value was $54 \mathrm{~Gy} \mathrm{~cm}^{2}$, while the maximum and minimum KAP values were 137.1 and $4.2 \mathrm{~Gy} \mathrm{~cm}^{2}$, respectively. The mean KAP values for adult procedures were $65 \mathrm{~Gy} \mathrm{~cm} \mathrm{~cm}^{2}$ for coronary

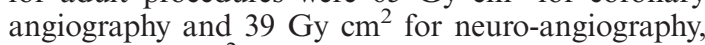
and $36 \mathrm{~Gy} \mathrm{~cm}^{2}$ for paediatric diagnostic cardiac

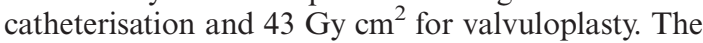


G. K. KORIR ET AL.

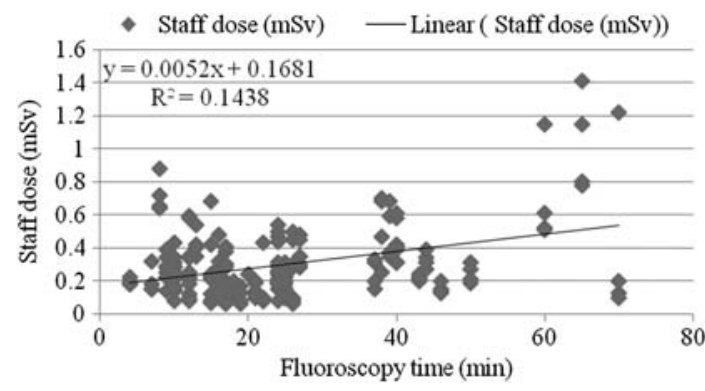

Figure 4. The relation between the occupational (staff) dose and fluoroscopy time.

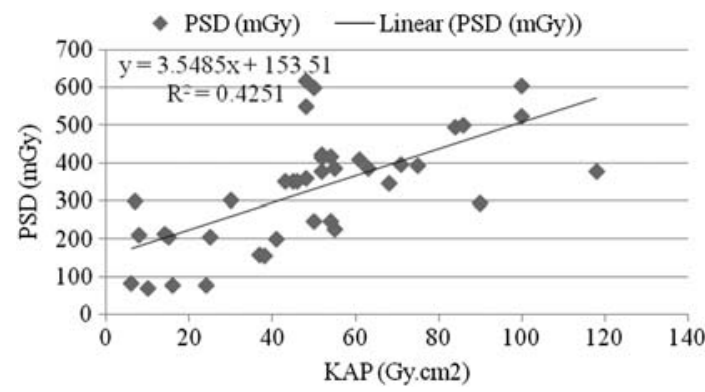

Figure 5. The correlation between PSD and KAP measurements.

fluoroscopic time ranged between 3 and 70 min with a mean of $30 \mathrm{~min}$. There was a KAP and fluoroscopy time correlation of $R^{2}=0.35$. This low correlation is due to the KAP dependence on the additional factors associated with the equipment such as the number of frames, $\mathrm{kVp}, \mathrm{mA}$, beam filtration and collimation. The initial KAP NDRLs per examination were $50 \mathrm{~Gy} \mathrm{~cm}^{2}$ for diagnostic cardiac catheterisation and $60 \mathrm{~Gy} \mathrm{~cm}^{2}$ for valvuloplasty in the case of paediatric cases, and $90 \mathrm{~Gy} \mathrm{~cm}^{2}$ for coronary angiography and $105 \mathrm{~Gy} \mathrm{~cm}^{2}$ for neuroangiography in the case of adults. The respective fluoroscopy time values in minutes were 32 and 40 for paediatric cases, while 26 and 20 were found in adult cases. The only available reference level was the KAP value of $45 \mathrm{~Gy} \mathrm{~cm}^{2}$ for adult coronary angiography ${ }^{(11)}$. The mean and third quartile values obtained in this study were above this reference level.

Figure 3 shows the distribution of the PSD and fluoroscopy time for the examinations considered in the study. The measured mean PSD was $347 \mathrm{mGy}$ (range 52-740 mGy). The fluoroscopic time and the patient's PSD were correlated with $R^{2}=0.49$. The mean PSDs per examination were $310 \mathrm{mGy}$ for diagnostic cardiac catheterisation and $397 \mathrm{mGy}$ for valvuloplasty for paediatric cases, and $435 \mathrm{mGy}$ for coronary angiography and $354 \mathrm{mGy}$ for neuro-angiography for adults.

\section{Occupational (staff) dose}

Figure 4 shows the distribution of the occupational dose and fluoroscopic time. The mean occupational dose in decreasing order was found to be as follows: MDs $0.4 \mathrm{mSv}$, nurses $0.31 \mathrm{mSv}$, clinical staff 0.27 $\mathrm{mSv}$ and technologists $0.25 \mathrm{mSv}$.

The linear correlation between the PSD and KAP values is shown in Figure 5. The low correlation may improve with fewer angulations (projections) and fewer variations in the image intensifier size.

\section{DISCUSSION}

In this study, $25 \%$ of IPs carried out in paediatric patients was higher than the figures reported in the International Atomic Energy Agency studies, where developed countries carried out at $10 \%$ and developing countries at $5 \%{ }^{(12)}$. The $75 \%$ adult IPs is also lower than $90 \%$, which has been reported for the developed countries, and $95 \%$, which has been reported for other developing countries in Africa. This could be due to the increasing number of incidences of heart diseases in paediatric patients and moderately increasing number of heart diseases in adults referred to in the study. One of the hospitals had the highest number and the widest range of procedures. However, the majority were diagnostic procedures, which comparatively, were low-dose procedures. Two other hospitals had the same machine model; hence, the user training given to the staff at the commissioning was likely to have been based on the same curriculum and patient imaging protocols. Three of the four hospitals had similarity in patient workload and average PSD values.

The low correlation between KAP and fluoroscopy time measurements indicates the need to study all the patient dose-dependent factors in radiation protection. The general linear relationship between the PSD and KAP shows that there was less variation in the projections or in the size of the image intensifier. The correlation is affected by differences in either the image intensifier size or the variation in the projections being so great such that increase in PSD did not correspond to that in KAP. Thus, for two similar KAP values obtained by the same staff and X-ray system, the skin dose does not show a similar correlation because of the uneven concentration of radiation fields on the patient's skin. The scatter diagram in Figure 5 shows more correlation towards PSD compared with KAP. Another research study has shown that KAP and PSD are independent quantities in practice, even for a given X-ray system or a specific procedure ${ }^{(13)}$. While PSD may be an indicator of deterministic effects, KAP may reliably estimate it 
with $R^{2}=0.49$. This correlation can be improved by increasing the size of a sample of patients to avoid large statistical variations of the mean value of the measured quantity. This may also improve the estimation of potential stochastic effects. In the same token, a large sample size will improve the accuracy of fluoroscopy time as a measure of radiation dose or an indicator of the potential radiation effects. The larger patient dose as well as initial NDRLs values obtained in this study is associated with the efficiency of the equipment, complexity of the procedure, clinical technique and the operator's experience of using the equipment.

The occupational doses measured for the groups of radiation workers normally present during interventional procedures indicate trends varying from hospital to hospital. In all the participating hospitals, it was evident that the most vulnerable group was the MDs who received an average of $0.34 \mathrm{mSv}$ per procedure. This is because of performing the interventions while standing near the radiation source and next to the patient. The value obtained in this study was above $0.3 \mu \mathrm{Sv}$, which has been reported for cardiologists, but correlated well with the $10-30 \mathrm{mSv}$ month $^{-1}$ reported in the literature ${ }^{(14)}$. The decreasing trend for the other staff was as follows: the nurses 0.27 $\mathrm{mSv}$, clinical staff $0.23 \mathrm{mSv}$ and the radiographers $0.22 \mathrm{mSv}$, respectively, per procedure. Considering the workload and the fact that some of the staff work in more than one facility, these values are above those obtained in a study conducted in the UK where interventional radiologists received an average annual dose of $0.35 \mathrm{mSv}$ and cardiologists $0.2 \mathrm{mSv} \mathrm{v}^{(15)}$. For both of these groups, almost $95 \%$ of the staff receives annual doses of $<1 \mathrm{mSv}$. The other occupational groups receive lower average annual doses. With the average exposure of $125 \mathrm{mSv} \mathrm{y}^{-1}$ and the number of patients increasing as indicated in this study, there is likelihood for the ICRP annual eye lens dose equivalent limit of $150 \mathrm{mSv} \mathrm{y}^{-1}$ to be exceeded, with the prevailing radiation safety practices ${ }^{(9)}$. The use of lead goggles and ceiling-suspended lead glass to reduce the exposure to the lens of the eye is inevitable. The condition of the machines and the level of training and experience of the staff are some of the likely contributors to both patient and occupational doses. It was also noted that owing to expertise, some cardiologists and radiologists carry out these procedures in more than one hospital but their monthly monitoring is done only at their primary centres. In addition, some staff either did not wear their TLD badges properly, regularly or did not wear them at all.

\section{CONCLUSIONS AND RECOMMENDATIONS}

Doctors participating in IPs receive the highest occupational radiation exposure. This external radiation exposure may exceed the ICRP annual eye lens dose equivalent limit because of the observed and increasing number of patients. This justifies the need for effective radiation safety practices that include adequate use of protective tools such as lead goggles and ceiling-suspended lead glass. Additionally, adequate personnel monitoring programmes relevant to personnel in the catheterisation laboratories should be established. This may include individual monitoring devices being worn, both under the protective apron at the waist and outside the protective apron at the neck.

The measured PSDs were within the threshold limit of 2 Gy per procedure for skin injuries. The patient records in IPs should be updated with patient dose, measurement and appropriate technical factors associated with the equipment. Personnel training on the appropriate use of equipment to practically manage radiation dose exposure levels should be enhanced in order to protect both patients and staff.

A code of practice to govern interventional procedures in the country should be developed with the input of all the hospitals involved, regulatory authorities and other relevant stakeholders to ensure safety in the practice. In addition, a follow-up study is necessary in order to monitor and evaluate the safety status periodically.

\section{ACKNOWLEDGEMENTS}

The authors sincerely thank the management and the staff of the four hospitals for their participation in the IAEA project (RAF/9/033-Strengthening Radiological Protection of Patient and Medical Exposure Control), the National Council for Science and Technology and the International Atomic Energy Agency.

\section{REFERENCES}

1. Taylor, W. and Rodesch, G. Interventional neuroradiology. Br. Med. J. 311, 789-792 (1995).

2. T-Rays. History of Fluoroscopy. Available on http:// taniagraphy.blogspot.com/2009/04/history-of-fluoroscopy. html.

3. Prieto, C., Vano, E., Fernandez, J. M., Galvan, C., Sabate, M., Gonzalez, L. and Martinez, D. Six years experience in intracoronary branchytherapy procedures: patient doses from fluoroscopy. Br. J. Radiol. 79, 730-733 (2006).

4. Ciraj-Bjelac, O., Rehani, M. M., Sim, K. H., Liew, H. B., Vano, E. and Kleiman, N. J. Cataract for staff in interventional cardiology: Is there reason for concern? Catheter. Cardiovasc. Interv. 76, 826-834 (2010).

5. International Commission on Radiation Protection. Avoidance of radiation injuries from medical interventional procedures. ICRP Publication 85. Ann. ICRP 30(2). Pergamon Press (2000).

6. Editorial. British Journal of Radiology Review of the year-2009. Br. J. Radiol. 83, 185-190 (2010). 


\section{G. K. KORIR ET AL.}

7. Miller, D. L., Balter, S., Schueler, B., Wagner, L. K. and Vano, E. Clinical radiation management for fluoroscopically guided interventional procedures. Radiology 257, 321-332 (2010).

8. Hart, D., Hillier, M. C. and Wall, B. F. National reference doses for common radiographic, fluoroscopic and dental $X$-ray examinations in the $U K$. Br. J. Radiol. 82, $1-12$ (2009).

9. International Commission on Radiological Protection. Recommendations of the International Commission on Radiological Protection. ICRP Publication 103. Ann. ICRP 37(2-4). Pergamon Press (2007).

10. Morrell, R.E. and Rogers, A. Calibration of Kodak EDR2 film for patient skin dose assessment in cardiac catheterization procedures. Phys. Med. Biol. 49, 55-59 (2004).

11. Padovani, R. et al. Reference levels at European level for cardiac interventional procedures.
Radiat. Prot. Dosim. 2008;129:104-107 (European Commission).

12. Virginia, T. et al. Radiation exposure to patients during interventional procedures in 20 countries: initial IAEA project results. Am. J. Roentgenol. 193, 559-569 (2009).

13. Vano, E., Gonzalez, L., Ten, J. I., Fernadez, J. M., Guibelalde, E. and Macaya, C. Skin dose and dosearea product values for interventional cardiology procedures. Br. J. Radiol. 74, 48-55 (2001).

14. Tsapaki, V. Occupational dose constraints in interventional cardiology procedures: the DIMOND approach. Phys. Med. Biol. 49, 997-1005 (2009).

15. Watson, S. J., Jones, A. L., Oatway, W. B. and Hughes, J. S. Ionizing radiation exposure of the UK population, 2005 review. Report HPA-RPD-001. Health Protection Agency-Radiation Protection Division (2005). 